\title{
Pullback Exponential Attractor for Second Order Nonautonomous Lattice System
}

\author{
Shengfan Zhou, ${ }^{1}$ Hong Chen, ${ }^{1}$ and Zhaojuan Wang ${ }^{2}$ \\ ${ }^{1}$ Department of Mathematics, Zhejiang Normal University, Jinhua 321004, China \\ ${ }^{2}$ School of Mathematical Science, Huaiyin Normal University, Huaiyin 223300, China \\ Correspondence should be addressed to Shengfan Zhou; zhoushengfan@yahoo.com
}

Received 7 January 2014; Accepted 27 February 2014; Published 3 April 2014

Academic Editor: Zengji Du

Copyright (c) 2014 Shengfan Zhou et al. This is an open access article distributed under the Creative Commons Attribution License, which permits unrestricted use, distribution, and reproduction in any medium, provided the original work is properly cited.

\begin{abstract}
We first present some sufficient conditions for the existence of a pullback exponential attractor for continuous process on the product space of the weighted spaces of infinite sequences. Then we prove the existence and continuity of a pullback exponential attractor for second order lattice system with time-dependent coupled coefficients in the weighted space of infinite sequences. Moreover, we obtain the upper bound of fractal dimension and attracting rate for the attractor.
\end{abstract}

\section{Introduction}

Lattice dynamical systems (LDSs), which include coupled systems of infinite ordinary differential equations and coupled map lattices, have drawn more and more attention because these systems appear in various fields $[1,2]$. In recent years, global attractors, uniform attractors, pullback attractors (or kernel sections), and random attractor for autonomous, nonautonomous, and stochastic LDSs have been studied; see [3-12]. However, these attractors sometimes attract orbits at a relatively slow speed, so that it might take an unexpected long time to be reached. Besides, it is usually difficult to estimate the attracting rate in terms of physical parameters of the model. Appropriate alternatives are exponential attractors and pullback exponential attractors which contain the global attractors and pullback attractors and attract all bounded sets exponentially [13-20]. For LDSs, $[13,16,21]$ studied the existence of exponential attractors for first order, second order, and partly dissipative autonomous LDSs, respectively. Zhou and Han [20] presented some sufficient conditions for the existence of pullback exponential attractors for LDSs in $l_{\rho}^{2}$ and provided applications to first order and partly dissipative nonautonomous LDSs. As we know, there are no results on pullback exponential attractors for second order nonautonomous LDSs which have been studied extensively [4-7, 9-13, 21-23]. In this paper, we study the following second order nonautonomous LDSs:

$$
\begin{aligned}
\frac{d^{2} u_{i}}{d t^{2}}+\alpha \frac{d u_{i}}{d t}= & -\lambda_{i} u_{i}+\sum_{j=-q}^{q} \eta_{i, j}(t) u_{i+j} \\
& +f_{i}\left(u_{i}, t\right)+g_{i}(t), \quad i \in \mathbb{Z},
\end{aligned}
$$

where for $i \in \mathbb{Z}, u_{i} \in \mathbb{R}, g_{i} \in C(\mathbb{R}, \mathbb{R}) ; f_{i} \in C^{1}(\mathbb{R} \times \mathbb{R}$, $\mathbb{R}) ; \eta_{i, j}(t), j=-q, \ldots, q(q \in \mathbb{N})$ are locally integrable in $t$, and $\lambda_{i}, \alpha$ are positive constants. First we present some sufficient conditions for the existence of a pullback exponential attractor for a continuous process on the product space $\prod_{j=1}^{m} l_{\rho_{j}}^{p}$ of infinite sequences, which are direct results of [20]. Then we prove the existence of a pullback exponential attractor for system (1) in weighted space $l_{\rho}^{2} \times l_{\rho}^{2}$; moreover, we obtain the upper bound of fractal dimension and attracting rate for the attractor.

\section{Preliminaries}

In this section, we present some sufficient conditions for the existence of a pullback exponential attractor for a continuous process on the product space of $m$ weighted space of infinite sequences. 
Let $\rho_{j}: \mathbb{Z} \rightarrow \mathbb{R}_{+}$be positive-valued function such that $0<\rho_{j}(i)=\rho_{j, i} \leq M_{j 0}$, where $M_{j 0}$ are positive constants, $j=1, \ldots, m$, and let

$$
l_{\rho_{j}}^{p}=\left\{u=\left(u_{i}\right)_{i \in \mathbb{Z}}: \sum_{i \in \mathbb{Z}} \rho_{j, i}\left|u_{i}\right|^{p}<\infty, u_{i} \in \mathbb{R}\right\}, \quad p>1,
$$

which is Banach space with norm $\|u\|_{\rho_{j}}^{p}=\sum_{i \in \mathbb{Z}} \rho_{j, i}\left|u_{i}\right|^{p}$ for $u=\left(u_{i}\right)_{i \in \mathbb{Z}}, v=\left(v_{i}\right)_{i \in \mathbb{Z}} \in l_{\rho_{j}}^{p}$. Let $l_{\rho, m}^{p}=\prod_{j=1}^{m} l_{\rho_{j}}^{p}$ be a product space of $m$ spaces $l_{\rho_{j}}^{p}, j=1, \ldots, m$. Write

$$
l_{\rho_{j}, N}^{p}=\left\{u=\left(u_{i}\right)_{i \in \mathbb{Z}} \in l_{\rho_{j}}^{p}: u_{i}=0 \text { for }|i|>N\right\}, \quad N \in \mathbb{N} .
$$

Then $l_{\rho_{j}, N}^{p}$ is $(2 N+1)$-dimensional subspaces of $l_{\rho_{j}}^{p}$. Let $l_{\rho, m, N}^{p}=\prod_{j=1}^{m} l_{\rho_{j, N}}^{p}$ and define a bounded projection $P_{N}$ : $l_{\rho, m}^{p} \rightarrow l_{\rho, m, N}^{p}$ as follows: for $\varphi=\left(\varphi_{i}\right)_{i \in \mathbb{Z}}=\left(u_{i}^{(1)}, \ldots, u_{i}^{(m)}\right)_{i \in \mathbb{Z}}$, $u^{(j)}=\left(u_{i}^{(j)}\right)_{i \in \mathbb{Z}} \in l_{\rho_{j}}^{p}$,

$$
\left(P_{N} \varphi\right)_{i}= \begin{cases}\varphi_{i}, & |i| \leq N \\ 0, & |i|>N\end{cases}
$$

Consider a two-parameter continuous process on $E$ : $W(t, \tau): E \rightarrow E, t \geq \tau \in \mathbb{R}$, satisfying the following: $W(\tau, \tau)=I$, for all $\tau \in \mathbb{R} ; W(t, r) W(r, \tau)=W(t, \tau),-\infty<$ $\tau \leq r \leq t<\infty ;\left(t, \tau, \varphi_{\tau}\right) \rightarrow W(t, \tau) \varphi_{\tau}$ is continuous for $-\infty<\tau \leq t<\infty$.

Definition 1. A family $\{\mathscr{M}(t)\}_{t \in \mathbb{R}}$ of subsets of $l_{\rho, m}^{p}$ is called a pullback exponential attractor for the continuous process $\{W(t, \tau)\}_{t \geq \tau}$, if

(i) each $\mathscr{M}(t)(t \in \mathbb{R})$ is a compact set of $l_{\rho, m}^{p}$ and its fractal dimension is uniformly bounded in $t$; that is, $\sup _{t \in \mathbb{R}} \operatorname{dim}_{f} \mathscr{M}(t)<\infty$;

(ii) it is positively invariant; that is, $W(t, \tau) \mathscr{M}(\tau) \subset \mathscr{M}(t)$ for all $-\infty<\tau \leq t<\infty$;

(iii) there exist an exponent $\alpha>0$ and two positivevalued functions $Q, \mathscr{T}: \mathbb{R}_{+} \rightarrow \mathbb{R}_{+}$such that for any bounded set $B \subset l_{\rho, m}^{p}$

$$
\begin{gathered}
\mathrm{d}_{h}(W(t, \tau) B, \mathscr{M}(t)) \leq Q\left(\|B\|_{l_{\rho, m}^{p}}\right) e^{-\alpha(t-\tau)}, \quad \tau \in \mathbb{R}, \\
\tau+\mathscr{T}\left(\|B\|_{p_{p, m}^{p}}\right) \leq t<\infty,
\end{gathered}
$$

where " $\mathrm{d}_{h}(\cdot, \cdot)$ " is the Hausdorff semidistance between two subsets of $l_{\rho, m}^{p}$.

As a direct consequence of Theorem 2 of [20], we have the following theorem.

Theorem 2. Let $\{W(t, \tau)\}_{t \geq \tau}$ be a continuous process in $l_{\rho, m}^{p}$. Assume that there exists a uniformly bounded absorbing set $B_{0} \subset l_{\rho, m}^{p}$ for $\{W(t, \tau)\}_{t \geq \tau}$ : for any bounded set $B \subset l_{\rho, m}^{p}$, there exists a constant $T_{B} \geq 0$ such that $W(t, \tau) B \subseteq B_{0}$ for all $t \geq \tau+$ $T_{B}, \tau \in \mathbb{R}$. For any $t \in \mathbb{R}$, set $X(t)=\overline{U_{s \geq T_{B_{0}}} W(t, t-s) B_{0}} \subseteq B_{0}$. If

(A1) there exist $T^{*}>T_{B_{0}}$ and $L_{T^{*}}=L\left(T^{*}\right)>0$ such that, for every $\tau \in \mathbb{R}$, any $t \in\left[0, T^{*}\right]$, and $\varphi_{\tau}, \psi_{\tau} \in X(\tau)$,

$$
\left\|W(t+\tau, \tau) \varphi_{\tau}-W(t+\tau, \tau) \psi_{\tau}\right\|_{l_{p, m}^{p}} \leq L_{T^{*}}\left\|\varphi_{\tau}-\psi_{\tau}\right\|_{p_{p, m}^{p}} ;
$$

(A2) there exist a positive constant $\gamma \in[0,1 / 2)$ and a $(2 N+$ 1)m-dimensional bounded projection $P_{N}: l_{\rho, m}^{p} \rightarrow$ $l_{\rho, m, N}^{p}$ such that, for every $\tau \in \mathbb{R}$ and $\varphi_{\tau}, \psi_{\tau} \in X(\tau)$,

$$
\begin{aligned}
& \left\|\left(I-P_{N}\right)\left(W(t+\tau, \tau) \varphi_{\tau}-W(t+\tau, \tau) \psi_{\tau}\right)\right\|_{p_{p, m}^{p}} \\
& \leq \gamma\left\|\varphi_{\tau}-\psi_{\tau}\right\|_{l_{p, m}^{p}} .
\end{aligned}
$$

Then $\{W(t, \tau)\}_{t \geq \tau}$ possesses a pullback exponential attractor $\{\mathscr{M}(t)\}_{t \in R}$ satisfying

$$
\mathscr{M}(t) \subseteq X(t) \subseteq B_{0}, \quad \operatorname{dim}_{f} \mathscr{M}(t) \leq \frac{\ln N_{\theta}}{-\ln a_{\theta}},
$$

and, for any bounded set $B \subset l_{\rho, m}^{p}$,

$$
\begin{aligned}
\mathrm{d}_{h}(W(t, \tau) B, \mathscr{M}(t)) & \leq \frac{L_{T^{*}} \operatorname{Re}^{\alpha T_{B}}}{a_{\theta}^{2}} e^{-\alpha(t-\tau)}, \\
-\infty & <\tau+T_{B}<t \leq+\infty,
\end{aligned}
$$

where $N_{\theta}$ is the minimal number of closed balls of $E$ with radius $\theta$ covering the closed unit ball $B_{N}(0,1)$ of $l_{\rho, m, N}^{p}$ centered at 0 , and $a_{\theta}=2\left(\gamma+\theta L_{T^{*}}\right)$ with $0<\theta<(1-2 \gamma) / 2 L_{T^{*}}, \alpha=$ $-\ln \theta / T^{*}$, and $R=\sup _{\varphi \in B_{0}}\|\varphi\|_{l_{\rho, m}^{p}}$.

\section{Pullback Exponential Attractor for Second Order Nonautonomous Lattice System}

In this section, we study the existence of a pullback exponential attractor for the continuous processes associated with the second order nonautonomous lattice system (1). Note that system (1) with initial conditions can be written as a vector form

$$
\begin{gathered}
\ddot{u}+\alpha \dot{u}=-\lambda u+A(t) u+f(u, t)+g(t), \quad t>\tau, \tau \in R, \\
u(\tau)=\left(u_{i, \tau}\right)_{i \in Z}=u_{\tau}, \quad \dot{u}(\tau)=\left(\dot{u}_{i}(\tau)\right)_{i \in \mathbb{Z}}=\left(u_{1 i}, \tau\right)_{i \in \mathbb{Z}}=u_{1 \tau},
\end{gathered}
$$

where $u=\left(u_{i}\right)_{i \in \mathbb{Z}},(A(t) u)_{i}=\sum_{j=-q}^{q} \eta_{i, j}(t) u_{i+j}, \lambda u=$ $\left(\lambda_{i} u_{i}\right)_{i \in \mathbb{Z}}, f(u, t)=\left(f_{i}\left(u_{i}, t\right)\right)_{i \in \mathbb{Z}}, g(t)=\left(g_{i}(t)\right)_{i \in \mathbb{Z}}$.

Throughout this section, let $\rho$ be a positive weight function from $\mathbb{Z}$ to $\left(0, M_{0}\right) \subset \mathbb{R}^{+}$satisfying

(P0) $0<\rho(i) \leq M_{0}, \rho_{i}=\rho(i) \leq c_{0} \rho(i \pm 1), i \in \mathbb{Z}$, for some positive constants $M_{0}$ and $c_{0}$. 
Consider the weighted Hilbert space of infinite sequences

$$
l_{\rho}^{2}=\left\{u=\left(u_{i}\right)_{i} \in Z: \sum_{i \in Z} \rho_{i} u_{i}^{2}<\infty, u_{i} \in R\right\}
$$

endowed with inner product $(u, v)_{\rho}=\sum_{i \in Z} \rho_{i} u_{i} v_{i}$ and norm $\|u\|_{\rho}^{2}=(u, v)_{\rho}=\sum_{i \in Z} \rho_{i} u_{i}^{2}$ for $u=\left(u_{i}\right)_{i \in Z}, v=\left(v_{i}\right)_{i \in Z} \in$ $l_{\rho}^{2}$. For any $u, v \in l_{\rho}^{2}$, define an inner product on $l_{\rho}^{2}$ by $(u, v)_{\lambda, \rho}=\sum_{i \in Z} \rho_{i} \lambda_{i} u_{i} v_{i}$, then the norm $\|\cdot\|_{\lambda, \rho}$ include by $(\cdot, \cdot)_{\lambda, \rho}$ is equivalent to the norm $\|\cdot\|_{\rho}$ include by $(\cdot, \cdot)_{\rho}$. Let

$$
l_{\lambda, \rho}^{2}=\left(l_{\rho}^{2},(\cdot, \cdot)_{\lambda, \rho},\|\cdot\|_{\lambda, \rho}\right), \quad E=l_{\lambda, \rho}^{2} \times l_{\rho}^{2} .
$$

We make the following assumptions on $\lambda_{i}, \eta_{i, j}, f_{i}$, and $g_{i}$, for $i \in \mathbb{Z}, j=-q, \ldots, q$.

(H1) There exist two positive constants $\underline{\lambda}$ and $\bar{\lambda}$ such that

$$
0<\underline{\lambda} \leq \lambda_{i} \leq \bar{\lambda}<+\infty, \quad \forall i \in \mathbb{Z} .
$$

(H2) Let $\eta(t)=\left\{\sup \left|\eta_{i, j}(t)\right|: i \in \mathbb{Z}, j=-q \cdots q\right\}<\infty(q \in$ $\mathbb{N})$ satisfy the following:

$(\mathrm{H} 2 \mathrm{a}) \eta(t) \in L_{\mathrm{loc}}^{1}(\mathbb{R})$ in $t$

$(\mathrm{H} 2 \mathrm{~b})$ there exists a continuous positive valued function $Q: \mathbb{R}_{+} \rightarrow \mathbb{R}_{+}$such that

$$
\begin{array}{r}
\int_{t-l}^{t} \eta(s) d s \leq Q(l), \quad 0 \leq \lim _{l \rightarrow+\infty} \frac{1}{l} \int_{t-l}^{t} \eta(s) d s \leq \bar{\eta}<\infty, \\
\forall t \in \mathbb{R}, \quad l \in \mathbb{R}_{+},
\end{array}
$$

where $\bar{\eta}$ is a positive constant;

(H2c) there exist $I_{0} \in \mathbb{N}$ and $\eta_{0}>0$ such that

$$
\sup \left\{\left|\eta_{i, j}(t)\right|:|i|>I_{0}, j=-q \cdots q\right\} \leq \eta_{0}, \quad \forall t \in \mathbb{R} .
$$

(H3) For all $i \in \mathbb{Z}$, let $f_{i}$ satisfy the following:

(H3a) $f_{i}(x, t)$ is differentiable in $x$ and continuous in $t$;

(H3b) for any $i \in \mathbb{Z}, f_{i}(0, t)=0, \sup _{x, t \in \mathbb{R}}\left|f_{i}(x, t)\right| \leq$ $\beta_{i}(t)$

(H3c) there exist functions $\Gamma \in C\left(\mathbb{R}_{+} \times \mathbb{R}, \mathbb{R}_{+}\right)$and $\widetilde{\Gamma} \epsilon$ $C\left(\mathbb{R}_{+} \times \mathbb{R}_{+}, \mathbb{R}_{+}\right)$such that

$$
\sup _{\sqrt{\sqrt{\rho_{i}}|x| \leq r}}\left|f_{i}^{\prime}(x, t)\right| \leq \Gamma(r, t), \quad i \in \mathbb{Z}, t \in \mathbb{R}, r \in \mathbb{R}_{+} \text {, }
$$

$\Gamma(0, t)=0, \quad \int_{\tau}^{\tau+l} \Gamma^{2}(r, t) d t \leq \widetilde{\Gamma}(r, l), \quad \forall \tau \in \mathbb{R}, l \in \mathbb{R}_{+}$.

$$
\text { (H4) } g(t)=\left(g_{i}(t)\right)_{i \in \mathbb{Z}} \in \mathbf{G}, \beta(t)=\left(\beta_{i}(t)\right)_{i \in \mathbb{Z}} \in \mathbf{G} \text {, where }
$$

$$
\mathbf{G}=\left\{g \in C_{b}\left(\mathbb{R}, l_{\rho}^{2}\right): \forall \eta>0, \exists I(\eta) \in \mathbb{N}\right.
$$

$$
\text { such that } \left.\sup _{t \in \mathbb{R}} \sum_{|i|>I(\eta)} \rho_{i} g_{i}^{2}(t)<\eta\right\}
$$

and $\||g|\|_{\rho}=\sup _{t \in \mathbb{R}}\left\|g_{i}(t)\right\|_{\rho},\|\| \beta\|\|_{\rho}=\sup _{t \in \mathbb{R}}\left\|\beta_{i}(t)\right\|_{\rho}$, $C_{b}\left(\mathbb{R}, l_{\rho}^{2}\right)$ denotes the space of all continuous bounded functions from $\mathbb{R}$ into $l_{\rho}^{2}$.

Letting

$$
v=\dot{u}+\varepsilon_{0} u, \quad \varepsilon_{0}=\frac{\underline{\lambda} \alpha}{3 \underline{\lambda}+\alpha^{2}}, \quad \varphi=\left(\begin{array}{l}
u \\
v
\end{array}\right),
$$

then the system (10) is equivalent to the following evolution equation:

$$
\begin{gathered}
\dot{\varphi}+C(\varphi)=F(\varphi, t), \quad t>\tau, \tau \in \mathbb{R}, \\
\varphi(\tau)=\left(\begin{array}{c}
u_{\tau} \\
v_{\tau}
\end{array}\right)=\left(\begin{array}{c}
u_{\tau} \\
u_{1 \tau}+\varepsilon_{0} u_{\tau}
\end{array}\right),
\end{gathered}
$$

where

$$
\begin{gathered}
F(\varphi, t)=\left(\begin{array}{c}
0 \\
A(t) u+g(t)+f(u, t)
\end{array}\right), \\
C(\varphi)=\left(\begin{array}{c}
\varepsilon_{0} u-v \\
\lambda u-\varepsilon_{0}\left(\alpha-\varepsilon_{0}\right) u+\left(\alpha-\varepsilon_{0}\right) v
\end{array}\right) .
\end{gathered}
$$

Definition 3. The function $\varphi:[\tau, \tau+T) \rightarrow E(T>0)$ is called a mild solution of the following lattice differential equations:

$$
\frac{d \varphi(t)}{d t}=H(\varphi, t), \quad \varphi=\left(\varphi_{i}\right)_{i \in \mathbb{Z}}, \quad H=\left(H_{i}\right)_{i \in \mathbb{Z}},
$$

$t \geq \tau \in \mathbb{R}$,

if $\varphi \in C([\tau, \tau+T), E)$ and

$\varphi_{i}(t, \tau)=\varphi_{i}(\tau)+\int_{\tau}^{t} H_{i}(\varphi(s), s) d s, \quad i \in \mathbb{Z}, t \in[\tau, \tau+T)$.

Theorem 4. Assume that (PO) and (H1)-(H4) hold. Then for any fixed $\tau \in \mathbb{R}$ and any initial data $\varphi(\tau)=\left(u_{\tau}, v_{\tau}\right) \in$ $E$, problem (19) admits a unique mild solution $\varphi\left(\cdot, \tau ; \varphi_{\tau}\right) \in$ $C([\tau,+\infty), E)$ with $\varphi\left(\tau, \tau ; \varphi_{\tau}\right)=\varphi_{\tau}$ and $\varphi\left(\cdot, \tau ; \varphi_{\tau}\right)$ being continuous in $\varphi_{\tau} \in E$, and the mapping

$$
\begin{aligned}
U(t, \tau): \varphi(\tau) & =\left(u_{\tau}, v_{\tau}\right) \in E \longrightarrow \varphi(t, \tau) \\
& =(u(t, \tau), v(t, \tau)) \in E, \quad t \geq \tau, \tau \in \mathbb{R},
\end{aligned}
$$

generates a continuous process $\{U(t, \tau)\}_{t \geq \tau}$ on $E$.

Proof. Let

$$
\widetilde{F}(u, t)=A(t) u+f(u, t)+g(t),
$$

and then $\widetilde{F}(u, t)$ is continuous in $u$ and locally integrable in $t$ from $l_{\rho}^{2} \times \mathbb{R}$ into $l_{\rho}^{2}$. For any bounded set $B \subset E$ with $\sup _{\varphi \in B}\|\varphi\| \leq r$, let

$$
h_{B}(t)=\left((2 q+1) \eta(t)+\Gamma\left(\frac{r}{\lambda}, t\right)\right) r+\||| g \mid\| \geq 0, \quad t \in \mathbb{R} .
$$


Then for $\varphi=(u, v), \varphi^{(j)}=\left(u^{(j)}, u^{(j)}\right) \in B, j=1,2$, by (P0), (H1)-(H4), we have

$$
\begin{gathered}
\|F(\varphi, t)\|_{E} \leq h_{B}(t), \\
\left\|F\left(\varphi^{(1)}, t\right)-F\left(\varphi^{(2)}, t\right)\right\|_{E} \leq h_{B}(t) \cdot\left\|\varphi^{(1)}-\varphi^{(2)}\right\|_{E}, \quad t \in \mathbb{R}, \\
\int_{\tau}^{\tau+1} h_{B}(t) d t<\infty, \quad \forall \tau \in \mathbb{R} .
\end{gathered}
$$

By the approximating method [24], it follows that problem (19) possesses a unique local mild solution $\varphi\left(\cdot, \tau ; \varphi_{\tau}\right) \in$ $C\left(\left[\tau, T_{\max }\right), E\right)$. Similar to the proof of Theorem 5 below concerning the existence of a uniformly bounded absorbing set, we can prove that $T_{\max }=+\infty$.

In the following part of this section, we assume that (P0) and $(\mathrm{H} 1)-(\mathrm{H} 4)$ and

$$
\varepsilon_{0}>2 \widetilde{q}\left(\frac{1}{\underline{\lambda}}+1\right) \bar{\eta}, \quad \widetilde{q}=q+\sum_{k=0}^{q} c_{0}^{k}
$$

hold.

Theorem 5. Assume that (PO) and (H1)-(H4) hold. There exists a uniform bounded closed absorbing ball $B_{0}=B_{0}\left(0, r_{0}\right)$ $\subset E$ of $\{U(t, \tau)\}_{t \geq \tau}$ centered at 0 with radius $r_{0}$ (independent of $\tau, t)$ such that, for any bounded subset $B \subset E$, there exists $T_{B} \geq 0$ yielding $U(t+\tau, \tau) B \subseteq B_{0}$ for all $t \geq T_{B}$.

Proof. Let $\varphi(t)=\left(\begin{array}{l}u_{i}(t) \\ v_{i}(t)\end{array}\right)_{i \in \mathbb{Z}}$ be a mild solution of (19), $t \geq \tau$. Since the set of continuous functions is dense in $L_{\text {loc }}^{1}(\mathbb{R})$, by $(\mathrm{H} 2 \mathrm{a})$, there exist sequences of continuous functions $\eta_{i, j}^{m}(t)$, $m \in \mathbb{N}$, such that

$$
\begin{gathered}
\lim _{m \rightarrow+\infty} \int_{\tau}^{t}\left|\eta_{i, j}^{m}(s)-\eta_{i, j}(s)\right| d s=0, \\
\left|\eta_{i, j}^{m}(t)\right| \leq\left|\eta_{i, j}(t)\right| \leq|\eta(t)|, \quad \forall \tau, t \in \mathbb{R} .
\end{gathered}
$$

Consider the following lattice differential equations:

$$
\begin{gathered}
\dot{\varphi}^{m}+C\left(\varphi^{m}\right)=F^{m}\left(\varphi^{m}, t\right), \quad t>\tau, \\
\varphi^{m}(\tau)=\varphi_{\tau}=\left(\begin{array}{c}
u_{\tau} \\
v_{\tau}
\end{array}\right)=\left(\begin{array}{c}
u_{\tau} \\
u_{1 \tau}+\varepsilon_{0} u_{\tau}
\end{array}\right), \quad \tau \in \mathbb{R},
\end{gathered}
$$

where

$$
\begin{aligned}
& \varphi^{m}=\left(\begin{array}{c}
u^{m} \\
v^{m}
\end{array}\right), \\
& F^{m}\left(\varphi^{m}, t\right)=\left(\begin{array}{c}
0 \\
A^{m}(t) u^{m}+f\left(u^{m}, t\right)+g(t)
\end{array}\right), \\
& C\left(\varphi^{m}\right)=\left(\begin{array}{c}
\varepsilon_{0} u^{m}-v^{m} \\
\lambda u^{m}-\varepsilon_{0}\left(\alpha-\varepsilon_{0}\right) u^{m}+\left(\alpha-\varepsilon_{0}\right) v^{m}
\end{array}\right), \\
& \left(A_{m}(t) u^{m}\right)_{i}=\sum_{j=-q}^{q} \eta_{i, j}^{m}(t) u_{i+j}^{m} .
\end{aligned}
$$

Combining the continuity of $F^{m}\left(\varphi^{m}, t\right)$ in $t$ with the proof of Theorem 4 , (36) has a unique strong solution $\varphi^{m}\left(\cdot, \tau, \varphi_{\tau}\right)=$ $\left(u^{m}\left(\cdot, \tau, \varphi_{\tau}\right), v^{m}\left(\cdot, \tau, \varphi_{\tau}\right)\right) \in C([\tau,+\infty), E) \cap C^{1}((\tau,+\infty), E)$ satisfying

$$
\begin{array}{r}
\varphi_{i}^{m}(t)=\varphi_{\tau}+\int_{\tau}^{t}\left(F_{i}\left(\varphi_{i}^{m}(s), s\right)-C\left(\varphi_{i}^{m}(s)\right)_{i}\right) d s, \\
t \in[\tau, \tau+T] .
\end{array}
$$

Taking the inner product $(\cdot, \cdot)_{E}$ of $(29)$ with $\varphi^{m}(t)$, we obtain

$$
\frac{1}{2} \frac{d\left\|\varphi^{m}\right\|_{E}^{2}}{d t}+\left(C\left(\varphi^{m}\right), \varphi^{m}\right)_{E}=\left(F^{m}\left(\varphi^{m}, t\right), \varphi^{m}\right)_{E}
$$

By some computation, we have that

$$
\begin{aligned}
& \left(C\left(\varphi^{m}\right), \varphi^{m}\right)_{E} \geq \frac{\varepsilon_{0}}{2}\left\|\varphi^{m}\right\|_{E}^{2}+\frac{\alpha}{2}\left\|v^{m}\right\|_{\rho}^{2}, \\
& \left(F^{m}\left(\varphi^{m}\right), \varphi^{m}\right)_{E} \leq \frac{1}{\alpha}\left(\|\beta\|\left\|_{\rho}^{2}+\right\|\|g\|_{\rho}^{2}\right)+\frac{\alpha}{2}\left\|v^{m}\right\|_{\rho}^{2} \\
& +\frac{1}{2} \widetilde{q}\left(\frac{1}{\lambda}+1\right) \eta(t) \cdot\left\|\varphi^{m}\right\|_{E}^{2} .
\end{aligned}
$$

Then we have that, for $t \geq \tau$,

$$
\begin{aligned}
\frac{d\left\|\varphi^{m}(t)\right\|_{E}^{2}}{d t} \leq & \left(-\varepsilon_{0}+\tilde{q}\left(\frac{1}{\underline{\lambda}}+1\right) \eta(t)\right) \cdot\left\|\varphi^{m}\right\|_{E}^{2} \\
& +\frac{2}{\alpha}\left(\|\| \beta\|\|_{\rho}^{2}+\||| g\|_{\rho}^{2}\right) .
\end{aligned}
$$

Applying Gronwall's inequality to (35) on $[\tau, t](t \geq \tau)$, we obtain

$$
\begin{aligned}
\left\|\varphi^{m}(t)\right\|^{2} \leq & e^{-\varepsilon_{0}(t-\tau)+\int_{\tau}^{t} \tilde{q}((1 / \underline{\lambda})+1) \eta(s) d s}\left\|\varphi_{\tau}^{m}\right\|_{E}^{2} \\
& +\frac{2}{\alpha}\left(\|\| \beta\|\|_{\rho}^{2}+\|\| g \|_{\rho}^{2}\right) \\
& \times \int_{\tau}^{t} e^{-\varepsilon_{0}(t-s)+\int_{s}^{t} \tilde{q}((1 / \underline{\lambda})+1) \eta(r) d r} d s \doteq \chi^{2}(t, \tau) .
\end{aligned}
$$

It then follows that, for $T>0$,

$$
\begin{array}{r}
\left\|F_{i}^{m}\left(\varphi_{i}^{m}, t\right)-\left(C\left(\varphi^{m}\right)\right)_{i}\right\|_{\mathbb{R}^{2}} \leq \kappa(T, \tau), \\
t \in[\tau, \tau+T], \quad i \in \mathbb{Z},
\end{array}
$$

for some $\kappa(T, \tau)>0$ independent of $m$ and $i$, and then, for any $t, s \in[\tau, \tau+T), m \in \mathbb{N}$,

$$
\begin{aligned}
\left\|\varphi_{i}^{m}(t)-\varphi_{i}^{m}(s)\right\|_{\mathbb{R}^{2}} & =\int_{s}^{t}\left\|F_{i}^{m}\left(\varphi_{i}^{m}, s\right)-\left(C\left(\varphi^{m}\right)\right)_{i}\right\|_{\mathbb{R}^{2}} d s \\
& \leq \kappa(T, \tau) \cdot|t-s|
\end{aligned}
$$

implies the equicontinuity of $\left\{\varphi_{i}^{m}(t)\right\}_{m \in \mathbb{N}}, t \in[\tau, \tau+T]$. By the Arzela-Ascoli Theorem, there exists a convergent 
subsequence $\left\{\varphi_{i}^{m_{k}}(t)\right\}_{m \in \mathbb{N}}$ of $\left\{\varphi_{i}^{m}(t)\right\}_{m \in \mathbb{N}}, t \in[\tau, \tau+T]$, such that

$$
\begin{array}{r}
\lim _{k \rightarrow \infty} \varphi_{i}^{m_{k}}(t) \longrightarrow \bar{\varphi}_{i}(t)=\left(\bar{u}_{i}(t), \bar{v}_{i}(t)\right), \\
t \in[\tau, \tau+T], \quad i \in \mathbb{Z},
\end{array}
$$

and $\bar{\varphi}_{i}(t)$ is continuous in $t \in[\tau, \tau+T]$; moreover, by (36), $\left|\bar{\varphi}_{i}(t)\right| \leq \chi(t, \tau)$ for $t \in[\tau, \tau+T]$. By the Lebesgue Convergence Theorem, we have

$$
\lim _{m \rightarrow+\infty} \int_{\tau}^{t}\left|\eta_{i, j}^{m_{k}}(s) u_{i}^{m_{k}}(s)-\eta_{i, j}(s) \bar{u}_{i}(s)\right| d s=0 .
$$

Thus, by replacing $m$ with $m_{k}$ in (31) and letting $k \rightarrow+\infty$, we have

$$
\begin{array}{r}
\bar{\varphi}_{i}(t)=\varphi_{\tau}+\int_{\tau}^{t}\left(F_{i}(\bar{\varphi}(s), s)-\left(C(\bar{\varphi}(s))_{i}\right) d s,\right. \\
t \in[\tau, \tau+T] .
\end{array}
$$

By the uniqueness of the mild solutions of (19), we have $\bar{\varphi}_{i}(t)=\varphi_{i}(t)$ for $t \in[\tau, \tau+T]$. By replacing $m$ with $m_{k}$ and letting $k \rightarrow \infty$ in (36), we have that for, $t \geq \tau$,

$$
\|\varphi(t)\|_{E}^{2} \leq e^{-\varepsilon_{0}(t-\tau)+\widetilde{q}((1 / \underline{\lambda})+1) \int_{\tau}^{t} \eta(s) d s} \cdot\left\|\varphi_{\tau}\right\|_{E}^{2}+\frac{1}{2} r^{2}(t, \tau),
$$

where

$$
r^{2}(t, \tau)=\frac{4}{\alpha}\left(\|\| \beta\left\|\left.\right|_{\rho} ^{2}+\right\||| g \mid \|_{\rho}^{2}\right) \int_{\tau}^{t} e^{-\varepsilon_{0}(t-s)+\int_{s}^{t} \widetilde{q}((1 / \underline{\lambda})+1) \eta(r) d r} d s .
$$

By (H2b), there exists $T_{1}>0$ such that for, $s \geq T_{1}$ and $t \in \mathbb{R}$,

$$
\frac{1}{s} \int_{t-s}^{t} \eta(r) d r \leq \bar{\eta} .
$$

Let

$$
\widetilde{k}=\varepsilon_{0}-\tilde{q}\left(\frac{1}{\lambda}+1\right) \bar{\eta}>0,
$$

and then

$$
\begin{aligned}
\int_{\tau}^{t} e^{-\varepsilon_{0}(t-s)+\int_{s}^{t} \widetilde{q}((1 / \underline{\lambda})+1) \eta(r) d r} d s \\
\quad \leq \int_{0}^{+\infty} e^{-\varepsilon_{0} s+\int_{t-s}^{t} \widetilde{q}((1 / \underline{\lambda})+1) \eta(r) d r} d s \\
\quad=\left(\int_{0}^{T_{1}}+\int_{T_{1}}^{+\infty}\right) e^{-\varepsilon_{0} s+\int_{t-s}^{t} \widetilde{q}((1 / \underline{\lambda})+1) \eta(r) d r} d s \\
\quad \leq e^{\tilde{q}((1 / \underline{\lambda})+1) Q\left(T_{1}\right)} T_{1}+\int_{T_{1}}^{+\infty} e^{-\varepsilon_{0} s+\widetilde{q}((1 / \underline{\lambda})+1) \bar{\eta} s} \\
\quad=e^{\widetilde{q}((1 / \underline{\lambda})+1) Q\left(T_{1}\right)} T_{1}+\frac{1}{\widetilde{k}} e^{-\widetilde{k} T_{1}}<\infty .
\end{aligned}
$$

Thus, for any bounded subset $B$ of $E$ and $\varphi_{\tau} \in B$,

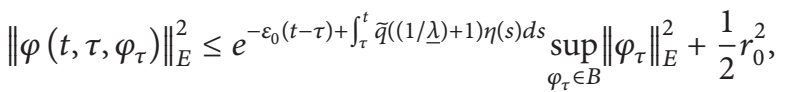

where

$$
\begin{aligned}
r_{0}^{2} & :=\frac{4}{\alpha}\left(\left\|\left|\beta\left\|_{\rho}^{2}+\right\|\|g\|\right|_{\rho}^{2}\right)\left(e^{\tilde{q}((1 / \underline{\lambda})+1) Q\left(T_{1}\right)} T_{1}+\frac{1}{\widetilde{k}} e^{-\tilde{k} T_{1}}\right)\right. \\
& <\infty .
\end{aligned}
$$

Again, by (H2b),

$$
\lim _{t \rightarrow+\infty} e^{-\varepsilon_{0}(t-\tau)+\int_{\tau}^{t} \widetilde{q}((1 / \underline{\lambda})+1) \eta(s) d s} \sup _{\varphi_{\tau} \in B}\left\|\varphi_{\tau}\right\|_{E}^{2}=0 .
$$

It then follows that

$$
B_{0}=\left\{\varphi \in E:\left\|\varphi_{\tau}\right\|_{E} \leq r_{0}\right\}=\overline{B_{E}\left(0, r_{0}\right)} \subset E
$$

is a uniformly bounded closed absorbing set for $\{U(t, \tau)\}_{t \geq \tau}$.

Lemma 6. Assume that (PO) and (H1)-(H4) hold. For any $\varepsilon>$ 0 , there exist $T\left(\varepsilon, B_{0}\right)>T_{B_{0}}$ and $I\left(\varepsilon, B_{0}\right) \in \mathbb{N}$ such that, for $t \geq T\left(\varepsilon, B_{0}\right)$, the mild solution $U(\tau+t, t) \varphi_{\tau}=\varphi\left(\tau+t, \tau ; \varphi_{\tau}\right)=$ $\left(u_{i}\left(\tau+t, \tau ; u_{\tau}\right), v_{i}\left(\tau+t, \tau ; v_{\tau}\right)\right)_{i \in \mathbb{Z}} \in$ E of system (19) with $\varphi_{\tau} \in$ $B_{0} \subset E$ satisfies

$$
\begin{aligned}
& \sum_{|i|>I\left(\varepsilon, B_{0}\right)}\left\|\varphi_{i}\left(\tau+t, \tau, \varphi_{\tau}\right)\right\|_{E}^{2} \\
& \quad=\sum_{|i|>I\left(\varepsilon, B_{0}\right)}\left(\lambda_{i} \rho_{i} u_{i}^{2}\left(\tau+t, \tau ; u_{\tau}\right)+\rho_{i} v_{i}^{2}\left(\tau+t, \tau ; v_{\tau}\right)\right) \\
& \quad \leq \varepsilon^{2} .
\end{aligned}
$$

Proof. Let $\xi \in C^{1}\left(R^{+}, R\right)$ be a smooth increasing function which satisfies

$$
\begin{gathered}
\xi(s)=0, \quad 0 \leq s<1 ; \\
0 \leq \xi(s) \leq 1, \quad 1 \leq s<2 ; \\
\xi(s)=1, \quad s \geq 2 ; \\
\left|\xi^{\prime}(s)\right| \leq C_{0}, \quad s \in R^{+}, C_{0}>0 .
\end{gathered}
$$

Let $\varphi^{m}(t)=\left(u_{i}^{m}, v_{i}^{m}\right)$ be a solution of (29) with $\varphi^{m}(\tau)=\varphi_{\tau}$. Let $M \geq I_{0}+q\left(I_{0}\right.$ is in (H2c)) be a suitable large integer, and set

$$
\begin{gathered}
x_{i}^{m}=\xi\left(\frac{|i|}{M}\right) u_{i}^{m}, \quad y_{i}^{m}=\xi\left(\frac{|i|}{M}\right) v_{i}^{m}, \\
z^{m}=\left(x^{m}, y^{m}\right)=\left(x_{i}^{m}, y_{i}^{m}\right)_{i \in \mathbb{Z}} .
\end{gathered}
$$


Taking the inner product $(\cdot, \cdot)_{E}$ of $(29)$ with $z^{m}$, we have

$$
\begin{aligned}
& \frac{1}{2} \frac{d}{d t} \sum_{i \in \mathbb{Z}} \xi\left(\frac{|i|}{M}\right)\left\|\varphi_{i}^{m}\right\|_{E}^{2} \\
& \quad+\sum_{i \in \mathbb{Z}} \xi\left(\frac{|i|}{M}\right)\left(\frac{\varepsilon_{0}}{2}\left\|\varphi_{i}^{m}\right\|_{E}^{2}+\frac{\alpha}{2}\left\|v_{i}^{m}\right\|_{\rho}^{2}\right) \\
& \leq\left(F^{m}\left(\varphi^{m}, t\right), z^{m}\right)_{E} .
\end{aligned}
$$

And we have the following estimates:

$$
\begin{gathered}
\left(f\left(u^{m}, t\right), y^{m}\right)_{\rho} \\
=\sum_{i \in \mathbb{Z}} \xi\left(\frac{|i|}{M}\right) \rho_{i} f_{i}\left(u_{i}^{m}, t\right) v_{i}^{m} \\
\leq \frac{1}{\alpha} \sum_{i \in \mathbb{Z}} \xi\left(\frac{|i|}{M}\right) \rho_{i} \beta_{i}^{2}(t)+\frac{\alpha}{4} \sum_{i \in \mathbb{Z}} \xi\left(\frac{|i|}{M}\right) \rho_{i}\left(v_{i}^{m}\right)^{2} \\
\leq \frac{1}{\alpha} \sum_{|i| \geq M} \rho_{i} \beta_{i}^{2}(t)+\frac{\alpha}{4} \sum_{i \in \mathbb{Z}} \xi\left(\frac{|i|}{M}\right)\left\|v_{i}^{m}\right\|_{\rho}^{2}, \\
\left(g(t), y^{m}\right)_{\rho} \leq \frac{1}{\alpha} \sum_{|i| \geq M} \rho_{i} g_{i}^{2}(t)+\frac{\alpha}{4} \sum_{i \in \mathbb{Z}} \xi\left(\frac{|i|}{M}\right)\left\|v_{i}^{m}\right\|_{\rho}^{2}, \\
\left(A_{m}(t) u^{m}, y^{m}\right)_{\rho} \\
\leq \frac{1}{2} \tilde{q}\left(\frac{1}{\lambda}+1\right) \eta(t) \sum_{i \in \mathbb{Z}} \xi\left(\frac{|i|}{M}\right)\left\|\varphi_{i}^{m}\right\|_{E}^{2}+\frac{k_{1}}{2 \underline{\lambda M}}\left\|\varphi^{m}\right\|_{E}^{2},
\end{gathered}
$$

where

$$
k_{1}=\eta_{0} C_{0}\left[q c_{0}^{q}+(q-1) c_{0}^{q}+\cdots+c_{0}\right]
$$

Therefore,

$$
\begin{aligned}
\frac{d}{d t} \sum_{i \in \mathbb{Z}} \xi\left(\frac{|i|}{M}\right)\left\|\varphi_{i}^{m}\right\|_{E}^{2} & \\
\leq & \left(-\varepsilon_{0}+\widetilde{q}\left(\frac{1}{\lambda}+1\right) \eta(t)\right) \sum_{i \in \mathbb{Z}} \xi\left(\frac{|i|}{M}\right)\left\|\varphi_{i}^{m}\right\|_{E}^{2} \\
& +\frac{2}{\alpha} \sum_{i \geq M} \rho_{i}\left(g_{i}^{2}(t)+\beta_{i}^{2}(t)\right)+\frac{k_{1}}{\underline{\lambda} M}\left\|\varphi^{m}\right\|_{E}^{2} .
\end{aligned}
$$

Applying Gronwall's inequality to (57) on $\left[\tau+T_{B_{0}}, \tau+t\right](t \geq$ $\left.T_{B_{0}}\right)$, we have that, for $\varphi_{\tau} \in B_{0}$,

$$
\begin{aligned}
\sum_{i \in Z} \xi\left(\frac{|i|}{M}\right)\left\|\varphi_{i}^{m}\left(\tau+t, \tau ; \varphi_{\tau}\right)\right\|_{E}^{2} \\
\quad \leq e^{-\varepsilon_{0}\left(t-T_{B_{0}}\right)+\int_{\tau+T_{B_{0}}}^{\tau+t} \tilde{q}((1 / \underline{\lambda})+1) \eta(s) d s}
\end{aligned}
$$

$$
\begin{aligned}
& \cdot\left\|\varphi^{m}\left(\tau+T_{B_{0}}, \tau ; \varphi_{\tau}\right)\right\|_{E}^{2} \\
& +\int_{\tau+T_{B_{0}}}^{\tau+t} e^{-\varepsilon_{0}(t+\tau-r)+\int_{r}^{\tau+t}} \tilde{q}((1 / \underline{\lambda})+1) \eta(s) d s \\
& \times \sum_{i \geq M} \frac{2}{\alpha} \rho_{i}\left(g_{i}^{2}(r)+\beta_{i}^{2}(r)\right) d r \\
& +\frac{k_{1}}{\underline{\lambda} M} \int_{\tau+T_{B_{0}}}^{\tau+t} e^{-\varepsilon_{0}(t+\tau-r)+\int_{r}^{\tau+t}} \tilde{q}((1 / \underline{\lambda})+1) \eta(s) d s
\end{aligned}
$$

$$
\times\left\|\varphi^{m}\left(r, \tau ; \varphi_{\tau}\right)\right\|_{E}^{2} d r
$$

By (44)-(45), for $t-T_{B_{0}} \geq T_{1}$, we have

$$
\begin{gathered}
e^{-\varepsilon_{0}\left(t-T_{B_{0}}\right)+\int_{\tau+T_{B_{0}}}^{\tau+t} \tilde{q}((1 / \underline{\lambda})+1) \eta(s) d s}\left\|\varphi^{m}\left(\tau+T_{B_{0}}, \tau, \varphi_{\tau}\right)\right\|_{E}^{2} \\
\leq e^{-\widetilde{k}\left(t-T_{B_{0}}\right)}\left\|\varphi^{m}\left(\tau+T_{B_{0}}, \tau, \varphi_{\tau}\right)\right\|_{E}^{2} \longrightarrow 0,
\end{gathered}
$$

as $t \rightarrow+\infty$. This means that, for any $\varepsilon>0$, there exists $T_{2}\left(\varepsilon, B_{0}\right) \geq T_{B_{0}}$ such that when $t \geq T_{2}\left(\varepsilon, B_{0}\right)$,

$$
e^{-\varepsilon_{0}\left(t-T_{B_{0}}\right)+\int_{\tau+T_{B_{0}}}^{\tau+t} \tilde{q}((1 / \underline{\lambda})+1) \eta(s) d s} \cdot\left\|\varphi^{m}\left(\tau+T_{B_{0}}, \tau, \varphi_{\tau}\right)\right\|_{E}^{2} \leq \frac{\varepsilon^{2}}{3} .
$$

By $(t), \beta(t) \in \mathbf{G}$, and (H2b), there exists $I_{1}(\varepsilon) \in \mathbb{N}$ such that, for $M>I_{1}(\varepsilon)$,

$$
\begin{aligned}
& \int_{\tau+T_{B_{0}}}^{\tau+t} e^{-\varepsilon_{0}(t+\tau-r)+\int_{r}^{\tau+t}} \tilde{q}((1 / \underline{\lambda})+1) \eta(s) d s \\
& \quad \times \sum_{i \geq M} \frac{2}{\alpha} \rho_{i}\left(g_{i}^{2}(r)+\beta_{i}^{2}(r)\right) d r \leq \frac{\varepsilon^{2}}{3} .
\end{aligned}
$$

From (47),

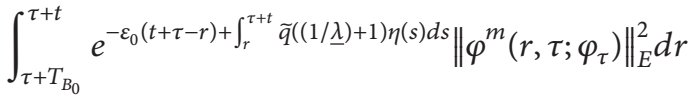

$$
\begin{aligned}
& \leq\left(t-T_{B_{0}}\right) e^{-\varepsilon_{0} t+\int_{0}^{t} \tilde{q}((1 / \underline{\lambda})+1) \eta(s) d s} \sup _{\varphi_{\tau} \in B_{0}}\left\|\varphi_{\tau}\right\|_{E}^{2}
\end{aligned}
$$

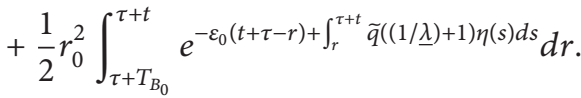

By (44), we have

$$
\begin{aligned}
& \left(t-T_{B_{0}}\right) e^{-\varepsilon_{0} t+\int_{0}^{t} \tilde{q}((1 / \underline{\lambda})+1) \eta(s) d s} \longrightarrow 0 \quad \text { as } t \longrightarrow+\infty,
\end{aligned}
$$

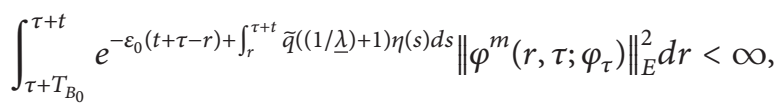

$$
\begin{aligned}
& t-T_{B_{0}} \geq T_{1} \text {. }
\end{aligned}
$$


And, for any $\varepsilon>0$, there exists $I_{2}\left(\varepsilon, B_{0}\right)>I_{0}+q$ such that, for $M>I_{2}\left(\varepsilon, B_{0}\right)$,

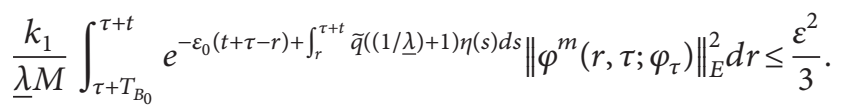

Let $T\left(\varepsilon, B_{0}\right)=\max \left\{T_{1}, T_{2}\left(\varepsilon, B_{0}\right)\right\}$ and $I\left(\varepsilon, B_{0}\right)=$ $2 \max \left\{I_{1}(\varepsilon), I_{2}\left(\varepsilon, B_{0}\right)\right.$. It follows immediately that

$$
\begin{array}{r}
\sum_{|i|>I\left(\varepsilon, B_{0}\right)}\left(\lambda_{i} \rho_{i} u_{i}^{2}\left(\tau+t, \tau ; u_{\tau}\right)+\rho_{i} v_{i}^{2}\left(\tau+t, \tau ; v_{\tau}\right)\right) \leq \varepsilon^{2} \\
t \geq T\left(\varepsilon, B_{0}\right) .
\end{array}
$$

In the following, we prove the existence of a pullback exponential attractor for $\{U(t, \tau)\}_{t \geq \tau}$ by applying Theorem 2 . For any $t \in \mathbb{R}$, set

$$
Y(t)=\overline{\bigcup_{s \geq T_{B_{0}}} U(t, t-s) B_{0}} \subseteq B_{0},
$$

where $B_{0}$ is the uniform absorbing set defined in Theorem 5 .

Theorem 7. Assume that (PO) and (H1)-(H4) hold.

(a) There exists a continuous positive value function $L_{t}$ in $t$ such that, for every $\tau \in \mathbb{R}$,

$$
\begin{array}{r}
\left\|U(t+\tau, \tau) \varphi_{\tau}-U(t+\tau, \tau) \psi_{\tau}\right\|_{E} \leq L_{t} \cdot\left\|\varphi_{\tau}-\psi_{\tau}\right\|_{E}, \\
\forall \varphi_{\tau}, \psi_{\tau} \in Y(\tau) .
\end{array}
$$

(b) There exist positive constants $T^{*}>0, \gamma \in[0,1 / 2)$, and a $2(2 N+1)$-dimensional orthogonal projection $P_{N}$ : $E \rightarrow E_{N}(N \in \mathbb{Z})$, such that, for every $\tau \in \mathbb{R}$ and $\varphi_{\tau}, \psi_{\tau} \in Y(\tau)$

$$
\begin{aligned}
& \left\|\left(I-P_{N}\right)\left(U\left(\tau+T^{*}, \tau\right) \varphi_{\tau}-U\left(\tau+T^{*}, \tau\right) \psi_{\tau}\right)\right\|_{E} \\
& \leq \gamma\left\|\varphi_{\tau}-\psi_{\tau}\right\|_{E} .
\end{aligned}
$$

(c) For each fixed $t \in \mathbb{R}, \quad \lim _{t \rightarrow \tau} \sup _{\varphi_{\tau} \in Y(\tau)}$ $\left\|U(t, \tau) \varphi_{\tau}-\varphi_{\tau}\right\|_{E}=0$.

Proof. For any $\tau \in \mathbb{R}$, initial data $\varphi_{\tau}=\left(u_{\tau}, v_{\tau}\right), \psi_{\tau}=\left(x_{\tau}, y_{\tau}\right)$. Let $\varphi(t)=U(t, \tau) \varphi_{\tau}, \psi(t)=U(t, \tau) \psi_{\tau}$, and $\phi(t)=\varphi(t)-$ $\psi(t)=\left(\phi_{i}(t)\right)_{i \in \mathbb{Z}}$; then, $\varphi(t), \psi(t), \phi(t) \in C([\tau,+\infty), E)$.

(a) For any $t \geq \tau$, let $\varphi^{m}(t), \psi^{m}(t)$ be two solutions of (29) with initial data $\varphi_{\tau}, \psi_{\tau}$ and set $\phi^{m}(t)=\varphi^{m}(t)-\psi^{m}(t)$, and then $\phi^{m}(t) \in C([\tau,+\infty), E) \cap C^{1}((\tau,+\infty), E)$ satisfies

$$
\begin{gathered}
\dot{\phi}^{m}+C\left(\phi^{m}\right)=F^{m}\left(\varphi^{m}, t\right)-F^{m}\left(\psi^{m}, t\right), \\
\phi^{m}(\tau)=\varphi_{\tau}-\psi_{\tau} \in E, \quad t>\tau .
\end{gathered}
$$

Taking the inner product $(\cdot, \cdot)_{E}$ of $(69)$ with $\phi^{m}$, we have

$$
\begin{aligned}
\frac{1}{2} \frac{d}{d t} \| & \phi^{m} \|_{E}^{2}+\left(C\left(\phi^{m}\right), \phi^{m}\right)_{E} \\
& =\left(F^{m}\left(\varphi^{m}, t\right)-F^{m}\left(\psi^{m}, t\right), \phi^{m}\right)_{E} .
\end{aligned}
$$

Since $\varphi_{\tau}, \psi_{\tau} \in Y(\tau)$, by (36) and (66), for $t \geq \tau$, $\varphi^{m}(t)=\left(u^{m}(t), v^{m}(t)\right), \psi^{m}(t)=\left(x^{m}(t), y^{m}(t)\right) \in$ $Y(t) \subseteq B_{0}$, thus $\left\|\varphi^{m}(t)\right\|_{E} \leq r_{0},\left\|\psi^{m}(t)\right\|_{E} \leq r_{0}$, and $\sup _{i \in \mathbb{Z}}\left\{\sqrt{\rho_{i}}\left|u_{i}^{m}(t)\right|, \sqrt{\rho_{i}}\left|x_{i}^{m}(t)\right|\right\} \leq r_{0} / \sqrt{\underline{\lambda}}$ for $t \geq \tau$. By (33), we have

$$
2\left(C\left(\phi^{m}\right), \phi^{m}\right)_{E} \geq \varepsilon_{0}\left\|\phi^{m}\right\|_{E}^{2}+\alpha\left\|v^{m}-y^{m}\right\|_{\rho}^{2},
$$

and, by (H2)-(H3),

$$
\begin{gathered}
2\left(A_{m}(t)\left(u^{m}-x^{m}\right), v^{m}-q^{m}\right)_{\rho} \leq \tilde{q}\left(\frac{1}{\underline{\lambda}}+1\right) \eta(t)\left\|\phi^{m}\right\|_{E}^{2}, \\
\left\|f\left(u^{m}, t\right)-f\left(p^{m}, t\right)\right\|_{\rho}^{2} \\
=\sum_{i \in \mathbb{Z}}\left[f_{i}^{\prime}\left(y_{i}^{m}+\vartheta_{i}\left(u_{i}^{m}-y_{i}^{m}\right), t\right)\right]^{2} \cdot\left(u_{i}^{m}-y_{i}^{m}\right)^{2} \\
\leq \Gamma^{2}\left(\frac{r_{0}}{\sqrt{\lambda}}, t\right)\left\|u^{m}-y^{m}\right\|_{\rho}^{2} \leq \frac{1}{\underline{\lambda}} \Gamma^{2}\left(\frac{r_{0}}{\sqrt{\lambda}}, t\right)\left\|\phi^{m}\right\|_{E}^{2}, \\
2\left(F^{m}\left(\varphi^{m}, t\right)-F^{m}\left(\psi^{m}, t\right), \phi^{m}\right)_{E} \\
\leq\left(\frac{1}{\alpha \underline{\lambda}} \Gamma^{2}\left(\frac{r_{0}}{\sqrt{\lambda}}, t\right)+\widetilde{q}\left(\frac{1}{\underline{\lambda}}+1\right) \eta(t)\right)\left\|\phi^{m}\right\|_{E}^{2} \\
+\alpha\left\|v^{m}-q^{m}\right\|_{\rho}^{2} .
\end{gathered}
$$

Thus,

$$
\begin{aligned}
& \frac{d}{d t}\left\|\phi^{m}(t)\right\|_{E}^{2} \\
& \quad \leq\left(-\varepsilon_{0}+\frac{1}{\alpha \underline{\lambda}} \Gamma^{2}\left(\frac{r_{0}}{\sqrt{\underline{\lambda}}}, t\right)+\widetilde{q}\left(\frac{1}{\underline{\lambda}}+1\right) \eta(t)\right) \cdot\left\|\phi^{m}(t)\right\|_{E}^{2} .
\end{aligned}
$$

Applying Gronwall's inequality to (73) on $[\tau, \tau+t],(t>0)$, we have

$$
\begin{gathered}
\left\|\phi^{m}(t+\tau)\right\|_{E}^{2} \leq e^{-\varepsilon_{0} t+\int_{\tau}^{\tau+t}\left((1 / \alpha \underline{\lambda}) \Gamma^{2}\left(\left(r_{0} / \sqrt{\underline{\lambda}}\right), s\right)+\widetilde{q}((1 / \underline{\lambda})+1) \eta(s)\right) d s} \\
\cdot\left\|\phi^{m}\left(\tau, \tau ; \phi_{\tau}\right)\right\|_{E}^{2} ;
\end{gathered}
$$

that is,

$$
\begin{aligned}
\left\|\phi^{m}(\tau+t)\right\|_{E} & =\left\|\varphi^{m}(\tau+t)-\psi^{m}(\tau+t)\right\|_{E} \\
& \leq L_{t} \cdot\left\|\varphi_{\tau}-\psi_{\tau}\right\|_{E}, \quad \forall t \geq 0,
\end{aligned}
$$

where

$$
L_{t}=e^{-\varepsilon_{0} t+\int_{\tau}^{\tau+t}\left((1 / \alpha \underline{\lambda}) \Gamma^{2}\left(\left(r_{0} / \sqrt{\underline{\lambda}}\right), s\right)+\widetilde{q}((1 / \underline{\lambda})+1) \eta(s)\right) d s}, \quad \forall t \geq 0 .
$$

By replacing $m$ with $m_{k}$ in (75) and letting $m_{k} \rightarrow \infty$, we have

$$
\left\|\varphi\left(\tau+t, \tau, \varphi_{\tau}\right)-\psi\left(\tau+t, \tau, \psi_{\tau}\right)\right\|_{E} \leq L_{t} \cdot\left\|\varphi_{\tau}-\psi_{\tau}\right\|_{E},
$$$$
\forall t \geq 0 \text {. }
$$ 
(b) For $i \in \mathbb{Z}$, let $\omega_{i}^{m}=\xi(|i| / M) \phi_{i}^{m}=(\xi(|i|) M)\left(u_{i}^{m}-\right.$ $\left.\left.x_{i}^{m}\right), \xi(|i| / M)\left(v_{i}^{m}-y_{i}^{m}\right)\right)$ and $\omega^{m}=\left(\omega_{i}^{m}\right)_{i \in \mathbb{Z}} \in E$, where $\xi$ is as in (52) and $M>I_{0}+q$. Taking the inner product of (69) with $\omega^{m}$ in $E$, we have that, for $t \geq \tau$,

$$
\begin{aligned}
& \frac{d}{d t} \sum_{i \in \mathbb{Z}} \xi\left(\frac{|i|}{M}\right)\left\|\phi_{i}^{m}\right\|_{E}^{2} \\
& \quad=-2\left(C\left(\phi^{m}\right), \omega^{m}\right)_{E}+2\left(F^{m}\left(\varphi^{m}, t\right)-F^{m}\left(\psi^{m}, t\right), \omega^{m}\right)_{E} .
\end{aligned}
$$

For the terms in the right hand of (78), we have

$$
\begin{gathered}
-2\left(C\left(\phi^{m}\right), \omega^{m}\right)_{E} \leq \sum_{i \in \mathbb{Z}} \xi\left(\frac{|i|}{M}\right)\left(-\varepsilon_{0}\left\|\phi_{i}^{m}\right\|_{E}^{2}-\alpha\left\|v_{i}^{m}-q_{i}^{m}\right\|_{\rho}^{2}\right), \\
2\left(A_{m}(t)\left(u^{m}-p^{m}\right), \bar{y}^{m}\right)_{\rho} \\
\leq \tilde{q}\left(\frac{1}{\underline{\lambda}}+1\right) \eta(t) \sum_{i \in \mathbb{Z}} \xi\left(\frac{|i|}{M}\right)\left\|\phi_{i}^{m}\right\|_{E}^{2}+\frac{k_{1}}{\underline{\lambda} M}\left\|\phi^{m}\right\|_{E}^{2},
\end{gathered}
$$

where

$$
\left\|\phi_{i}^{m}\right\|_{E}^{2}=\rho_{i}\left(\lambda_{i}\left|u_{i}^{m}\right|^{2}+\left|v_{i}^{m}\right|^{2}\right) \quad \text { for } \phi_{i}^{m}=\left(u_{i}^{m}, v_{i}^{m}\right) .
$$

By the continuity of $\Gamma(u, t)$ and $\Gamma(0, t)=0($ see $(\mathrm{H} 3 \mathrm{c}))$, there exists $\widetilde{\delta}$ such that

$$
\Gamma^{2}(\widetilde{\delta}, t) \leq \frac{\lambda \alpha \varepsilon_{0}}{2}, \quad t \in \mathbb{R} .
$$

By Lemma 6 and for $\widetilde{\delta}>0$, there exist $I(\widetilde{\delta}) \in \mathbb{N}, T_{2}\left(\widetilde{\delta}, B_{0}\right)>0$ such that, for $t \geq T_{2}\left(\widetilde{\delta}, B_{0}\right)$,

$$
\sup _{i>I(\widetilde{\delta})}\left\{\lambda_{i} \rho_{i}\left|u_{i}^{m}\left(t, \tau ; u_{\tau}\right)\right|^{2}, \lambda_{i} \rho_{i}\left|x_{i}^{m}\left(t, \tau ; p_{\tau}\right)\right|^{2}\right\} \leq \underline{\lambda} \widetilde{\delta}^{2},
$$

implying that, for $|i|>I(\widetilde{\delta})$,

and thus

$$
\sqrt{\rho_{i}}\left|u_{i}^{m}+\theta_{i}^{m}\left(u_{i}^{m}-p_{i}^{m}\right)\right| \leq \widetilde{\delta}, \quad \text { for } \theta_{i} \in(0,1),
$$

$$
\sup _{|i|>I(\widetilde{\delta})}\left|f_{i}^{\prime}\left(u_{i}^{m}+\theta_{i}^{m}\left(p_{i}^{m}-u_{i}^{m}\right), t\right)\right|^{2} \leq \Gamma^{2}(\widetilde{\delta}, t) \leq \frac{\lambda \alpha \varepsilon_{0}}{2},
$$

$t \in \mathbb{R}$
For $|i| \geq M \geq I(\widetilde{\delta})$ and $t>T_{2}\left(\widetilde{\delta}, B_{0}\right)>T_{1}$,

$$
\begin{aligned}
& 2\left(f\left(u^{m}, t\right)-f\left(x^{m}, t\right), \bar{y}^{m}\right)_{\rho} \\
& \leq \sum_{i \in \mathbb{Z}} \xi\left(\frac{|i|}{M}\right) \rho_{i}\left(\frac{1}{\alpha}\left|f^{\prime}\left(u_{i}^{m}+\theta_{i}^{m}\left(y_{i}^{m}-u_{i}^{m}\right)\right)\right|^{2}\right. \\
& \left.\quad \times\left|u_{i}^{m}-y_{i}^{m}\right|^{2}+\alpha\left|v_{i}^{m}-q_{i}^{m}\right|^{2}\right) \\
& \leq \sum_{i \in \mathbb{Z}} \xi\left(\frac{|i|}{M}\right) \frac{1}{\lambda \alpha}\left|f^{\prime}\left(u_{i}^{m}+\theta_{i}^{m}\left(p_{i}^{m}-u_{i}^{m}\right)\right)\right|^{2}\left\|\phi_{i}^{m}\right\|_{E}^{2} \\
& \quad+\alpha \sum_{i \in \mathbb{Z}} \rho_{i}\left|v_{i}^{m}-y_{i}^{m}\right|^{2} \\
& \leq \frac{\varepsilon_{0}}{2} \sum_{i \in \mathbb{Z}} \xi\left(\frac{|i|}{M}\right)\left\|\phi_{i}^{m}\right\|_{E}^{2}+\alpha\left\|v^{m}-y^{m}\right\|_{\rho}^{2} .
\end{aligned}
$$

Therefore, we obtain that, for all $M \geq \max \left\{I(\widetilde{\delta}), I_{0}+q\right\}, t>$ $T_{2}\left(\widetilde{\delta}, B_{0}\right)$,

$$
\begin{aligned}
\frac{d}{d t} \sum_{i \in \mathbb{Z}} \xi\left(\frac{|i|}{M}\right)\left\|\phi_{i}^{m}\right\|_{E}^{2} & \\
\leq & \sum_{i \in \mathbb{Z}} \xi\left(\frac{|i|}{M}\right)\left(-\frac{\varepsilon_{0}}{2}+\tilde{q}\left(\frac{1}{\lambda}+1\right) \eta(t)\right)\left\|\phi_{i}^{m}\right\|_{E}^{2} \\
& +\frac{k_{1}}{\underline{\lambda} M}\left\|\phi^{m}\left(t, \tau ; \phi_{\tau}\right)\right\|_{E}^{2} .
\end{aligned}
$$

Applying Gronwall's inequality to (86) from $\tau+T_{2}\left(T_{2}=\right.$ $\left.T_{2}\left(\widetilde{\delta}, B_{0}\right)\right)$ to $\tau+t\left(t \geq T_{2}+T_{1}\right)$, we have

$$
\begin{aligned}
\sum_{i \in \mathbb{Z}} \xi\left(\frac{|i|}{M}\right)\left\|\phi_{i}^{m}(\tau+t)\right\|_{E}^{2} \\
\leq e^{-\left(\varepsilon_{0} / 2\right)\left(t-T_{2}\right)+\int_{\tau+T_{2}}^{\tau+t} \tilde{q}((1 / \underline{\lambda})+1) \eta(s) d s}\left\|\phi^{m}\left(\tau+T_{2}, \tau ; \phi_{\tau}\right)\right\|_{E}^{2} \\
+\frac{k_{1}}{\underline{\lambda} M} \int_{\tau+T_{2}}^{\tau+t} e^{-\left(\varepsilon_{0} / 2\right)(\tau+t-r)+\int_{r}^{\tau+t}} \tilde{q}((1 / \underline{\lambda})+1) \eta(s) d s \\
\quad \times\left\|\phi^{m}\left(r, \tau ; \phi_{\tau}\right)\right\|_{E}^{2} d r .
\end{aligned}
$$

Thus, for $t \geq T_{2}+T_{1}$, we have

$$
\begin{aligned}
& e^{-\left(\varepsilon_{0} / 2\right)\left(t-T_{2}\right)+\int_{\tau+T_{2}}^{\tau+t} \tilde{q}((1 / \underline{\lambda})+1) \eta(s) d s}\left\|\phi^{m}\left(\tau+T_{2}, \tau ; \phi_{\tau}\right)\right\|_{E}^{2} \\
& \quad \leq e^{-\left(\varepsilon_{0} / 2\right)\left(t-T_{2}\right)+\int_{\tau+T_{2}}^{\tau+t} \widetilde{q}((1 / \underline{\lambda})+1) \eta(s) d s-\varepsilon_{0} T_{2}+(1 / \alpha \underline{\lambda}) \widetilde{\Gamma}^{2}\left(\left(r_{0} / \sqrt{\underline{\lambda}}\right), T_{2}\right)+\widetilde{q}((1 / \underline{\lambda})+1) \int_{\tau}^{\tau+T_{2}} \eta(s) d s}\left\|\phi^{m}\left(\tau, \tau ; \phi_{\tau}\right)\right\|_{E}^{2} \\
& \quad \leq e^{-\widetilde{k} t-\varepsilon_{0} T_{2}+(1 / \alpha \underline{\lambda}) \widetilde{\Gamma}^{2}\left(\left(r_{0} / \sqrt{\underline{\lambda}}\right), T_{2}\right)+\widetilde{q}((1 / \underline{\lambda})+1) \bar{\eta} T_{2}} \cdot\left\|\phi^{m}\left(\tau, \tau ; \phi_{\tau}\right)\right\|_{E}^{2}=k_{2} e^{-\widetilde{k} t} \cdot\left\|\phi^{m}\left(\tau, \tau ; \phi_{\tau}\right)\right\|_{E}^{2},
\end{aligned}
$$


where

$$
\begin{aligned}
& k_{2}=e^{-\varepsilon_{0} T_{2}+(1 / \alpha \underline{\lambda}) \widetilde{\Gamma}^{2}\left(\left(r_{0} / \sqrt{\underline{\lambda}}\right), T_{2}\right)+\widetilde{q}((1 / \underline{\lambda})+1) \bar{\eta} T_{2}}<\infty,
\end{aligned}
$$

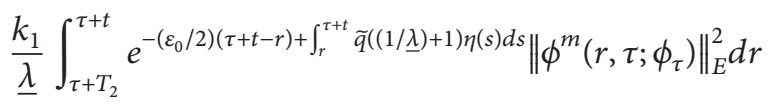

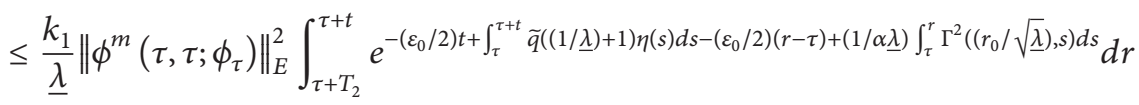

$$
\begin{aligned}
& \leq \frac{k_{1}}{\underline{\lambda}}\left\|\phi^{m}\left(\tau, \tau ; \phi_{\tau}\right)\right\|_{E}^{2} \int_{\tau+T_{2}}^{\tau+t} e^{-\left(\varepsilon_{0} / 2\right) t+\widetilde{q}((1 / \underline{\lambda})+1) \bar{\eta} t-\left(\varepsilon_{0} / 2\right)(r-\tau)+(1 / \alpha \underline{\lambda}) \widetilde{\Gamma}^{2}\left(\left(r_{0} / \sqrt{\lambda}\right), t\right)} d r \\
& \leq\left\|\phi^{m}\left(\tau, \tau ; \phi_{\tau}\right)\right\|_{E}^{2} \cdot \frac{k_{1}}{\underline{\lambda}} \frac{2}{\varepsilon_{0}} e^{-\left(\varepsilon_{0} / 2\right) t+\widetilde{q}((1 / \underline{\lambda})+1) \bar{\eta} t-\left(\varepsilon_{0} / 2\right) T_{2}+(1 / \alpha \underline{\lambda}) \widetilde{\Gamma}^{2}\left(\left(r_{0} / \sqrt{\lambda}\right), t\right)} \\
& =k_{3}(t) \cdot\left\|\phi^{m}\left(\tau, \tau ; \phi_{\tau}\right)\right\|_{E}^{2} \text {, }
\end{aligned}
$$

where

$$
k_{3}(t)=\frac{k_{1}}{\underline{\lambda}} \frac{2}{\varepsilon_{0}} e^{-\left(\varepsilon_{0} / 2\right) t+\tilde{q}((1 / \underline{\lambda})+1) \bar{\eta} t-\left(\varepsilon_{0} / 2\right) T_{2}+(1 / \alpha \underline{\lambda}) \tilde{\Gamma}^{2}\left(\left(r_{0} / \sqrt{\underline{\lambda}}\right), t\right)} .
$$

Thus, for $M \geq \max \left\{I(\widetilde{\delta}), I_{0}+q\right\}, t \geq T_{2}\left(\widetilde{\delta}, B_{0}\right)+T_{1}$, we have

$$
\begin{aligned}
\sum_{|i|>2 M}\left\|\phi_{i}^{m}(t+\tau)\right\|_{E}^{2} & \leq \sum_{i \in \mathbb{Z}} \xi\left(\frac{|i|}{M}\right)\left\|\phi_{i}^{m}(t+\tau)\right\|_{E}^{2} \\
& \leq\left(k_{2} e^{-\tilde{k} t}+\frac{1}{M} k_{3}(t)\right) \cdot\left\|\phi^{m}\left(\tau, \tau ; \phi_{\tau}\right)\right\|_{E}^{2} .
\end{aligned}
$$

Taking

$$
\begin{gathered}
T^{*}=\max \left\{T_{2}\left(\widetilde{\delta}, B_{0}\right)+T_{1}, \frac{\ln \left(8 k_{2}\right)}{k_{2}}\right\}, \\
N=\left\{2 I(\widetilde{\delta})+1,2 I_{0}+2 q, 8 k_{3}\left(T^{*}\right)\right\},
\end{gathered}
$$

then

$$
\gamma=\sqrt{k_{2} e^{-\tilde{k} T^{*}}+\frac{1}{N} k_{3}\left(T^{*}\right)}<\frac{1}{2} .
$$

Thus, by (91), we have

$$
\begin{aligned}
& \left\|\left(I-P_{N}\right)\left(U\left(\tau+T^{*}, \tau\right) \varphi_{\tau}-U\left(\tau+T^{*}, \tau\right) \psi_{\tau}\right)\right\|_{E} \\
& \leq \gamma\left\|\varphi_{\tau}-\psi_{\tau}\right\|_{E} .
\end{aligned}
$$

(c) It follows from the following estimate:

$$
\begin{aligned}
& \left\|U(t, \tau) \varphi_{\tau}-\varphi_{\tau}\right\|_{E}^{2} \\
& \quad \leq(t-\tau)\left(\int_{\tau}^{t}\|F(\varphi(s), s)-C(\varphi(s))\|_{E}^{2} d s\right)^{1 / 2}
\end{aligned}
$$

$$
\begin{gathered}
\leq(t-\tau) \int_{\tau}^{t}\left[k_{4}+3(2 q+1)^{2} r_{0}^{2} \eta^{2}(s)+r_{0}^{2} \Gamma^{2}\left(\frac{r_{0}}{\underline{\lambda}}, s\right)\right] d s \\
\leq(t-\tau)\left[k_{4}(t-\tau)+3(2 q+1)^{2} r_{0}^{2} \int_{\tau}^{t} \eta^{2}(s) d s\right. \\
\left.+r_{0}^{2} \int_{\tau}^{t} \Gamma^{2}\left(\frac{r_{0}}{\lambda}, s\right) d s\right] \\
\leq(t-\tau)\left[k_{4}(t-\tau)+3(2 q+1)^{2} r_{0}^{2} Q(t-\tau)\right. \\
\left.+r_{0}^{2} \widetilde{\Gamma}\left(\frac{r_{0}}{\underline{\lambda}}, t-\tau\right)\right] .
\end{gathered}
$$

and the continuity of $Q(\cdot), \widetilde{\Gamma}(\cdot, \cdot)$.

As a direct consequence of Theorem 2, Theorem 4, Theorem 5, Theorem 7, and Theorem 3.1 of [15], we have the following result.

Theorem 8. Assume that (PO) and (H1)-(H4) hold. The process $\{U(t, \tau)\}_{t \geq \tau}$ associated with (19) possesses a pullback exponential attractor $\{\mathscr{A}(t)\}_{t \in \mathbb{R}}$ with the following properties:

(i) for any $t \in \mathbb{R}, \mathscr{A}(t) \subseteq Y(t) \subseteq B_{0}$;

(ii) $\operatorname{dim}_{f} \mathscr{A}(t) \leq \ln N_{\theta}^{*} /-\ln a_{\theta}^{*}$;

(iii) and for any bounded set $B \subset E, d_{h}(U(t, \tau) Y(\tau)$, $\mathscr{A}(t)) \leq\left(L\left(T^{*}\right) r_{0} e^{\alpha^{*} T_{B}} /\left(a_{\theta}^{*}\right)^{2}\right) e^{-\alpha^{*}(t-\tau)},-\infty<\tau+T_{B}<$ $t \leq+\infty$;

(iv) $\lim _{t \rightarrow \tau} d_{h}(\mathscr{A}(t), \mathscr{A}(\tau))=0$;

where

$$
a_{\theta}^{*}=2\left(\gamma+\theta L_{T^{*}}\right), \quad \theta=\frac{1-2 \gamma}{4 L_{T^{*}}}, \quad \alpha^{*}=\frac{-\ln a_{\theta}^{*}}{T^{*}},
$$

and $N_{\theta}^{*}$ is the minimal number of closed balls of $E$ with radius $\theta$ covering the closed unit ball $B_{N}(0,1)$ of $E_{N}$ centered at 0 . 
Remark 9. It should be pointed out that, for some special cases, (H2) and (27) can be removed. For example, if the coupled linear operator $A(t) \equiv A$ in (10) is a constant operator $A$ satisfying $-A=D^{*} D=D D^{*}$, where $(D u)_{i}=$ $\sum_{j=-m_{0}}^{j=m_{0}} d_{j} u_{i+j}$, for all $u=\left(u_{i}\right)_{i \in \mathbb{Z}},\left|d_{j}\right| \leqslant \tilde{a}$ (constant), $-m_{0} \leqslant$ $j \leqslant m_{0}$, and $D^{*}$ is the adjoint of $D$. A simple example of such an operator $A$ is $(A u)_{i}=u_{i-1}+u_{i+1}-2 u_{i}$ and $(D u)_{i}=u_{i+1}-u_{i}$. Choose a positive weight function $\rho: \mathbb{Z} \rightarrow \mathbb{R}_{+}$satisfying the properties (P0) and $|\rho(i \pm 1)-\rho(i)| \leq b \rho(i)$, for all $i \in \mathbb{Z}$ for some small $b \geq 0$. Two examples of such weight functions are $\rho(i)=1 /\left(1+\kappa^{2} i^{2}\right)^{q}, q>1 / 2$, and $\rho(i)=e^{-\kappa|i|}, i \in \mathbb{Z}$, with $\kappa>0$ being a parameter. If the number $b$ satisfies

$$
0 \leq b<\min \left\{\frac{\varepsilon_{0}}{2 \widetilde{a} c_{2 m_{0}}}, \frac{\alpha}{4 \widetilde{a} c_{2 m_{0}}^{2}\left(2 m_{0}+1\right)^{2}}\right\},
$$

where $c_{2 m_{0}}=c_{0}^{m_{0}-1}+c_{0}^{m_{0}-2}+\cdots+c_{0}+1$, then conditions $(\mathrm{H} 2)$ and (27) can be removed.

\section{Conflict of Interests}

The authors declare that there is no conflict of interests regarding the publication of this paper.

\section{Acknowledgments}

This work is supported by the National Natural Science Foundation of China under Grant no. 11071165, 11326114 and Zhejiang Normal University (ZC304011068).

\section{References}

[1] H. Chate and M. Courbage, "Lattice systems," Physica D, vol. 10, no. 3-4, pp. 1-612, 1997.

[2] S.-N. Chow, "Lattice dynamical systems," in Dynamical Systems, vol. 1822 of Lecture Notes in Mathematics, pp. 1-102, Springer, Berlin, Germany, 2003.

[3] P. W. Bates, K. Lu, and B. Wang, "Attractors for lattice dynamical systems," International Journal of Bifurcation and Chaos in Applied Sciences and Engineering, vol. 11, no. 1, pp. 143-153, 2001.

[4] X. Fan and Y. Wang, "Attractors for a second order nonautonomous lattice dynamical system with nonlinear damping," Physics Letters A, vol. 365, no. 1-2, pp. 17-27, 2007.

[5] X. Han, "Random attractors for second order stochastic lattice dynamical systems with multiplicative noise in weighted spaces," Stochastics and Dynamics, vol. 12, no. 3, Article ID 1150024, 20 pages, 2012.

[6] X. Han, "Asymptotic behaviors for second order stochastic lattice dynamical systems on $\mathbb{Z}^{k}$ in weighted spaces," Journal of Mathematical Analysis and Applications, vol. 397, no. 1, pp. 242254, 2013.

[7] J. C. Oliveira, J. M. Pereira, and G. Perla Menzala, "Attractors for second order periodic lattices with nonlinear damping," Journal of Difference Equations and Applications, vol. 14, no. 9, pp. 899921, 2008.

[8] B. Wang, "Dynamics of systems on infinite lattices," Journal of Differential Equations, vol. 221, no. 1, pp. 224-245, 2006.
[9] C. Zhao and S. Zhou, "Upper semicontinuity of attractors for lattice systems under singular perturbations," Nonlinear Analysis. Theory, Methods \& Applications A, vol. 72, no. 5, pp. 2149-2158, 2010.

[10] X.-Q. Zhao and S. Zhou, "Kernel sections for processes and nonautonomous lattice systems," Discrete and Continuous Dynamical Systems B, vol. 9, no. 3-4, pp. 763-785, 2008.

[11] S. Zhou, "Attractors for second order lattice dynamical systems," Journal of Differential Equations, vol. 179, no. 2, pp. 605-624, 2002.

[12] S. Zhou and L. Wei, "A random attractor for a stochastic second order lattice system with random coupled coefficients," Journal of Mathematical Analysis and Applications, vol. 395, no. 1, pp. 42-55, 2012.

[13] A. Y. Abdallah, "Exponential attractors for second order lattice dynamical systems," Communications on Pure and Applied Analysis, vol. 8, no. 3, pp. 803-813, 2009.

[14] A. Eden, C. Foias, B. Nicolaenko, and R. Teman, Exponential Attractors for Dissipative Evolution Equation, Research in Applied Mathematics, vol. 37, Masson/John Wiley, Paris, France, 1994.

[15] M. Efendiev, Y. Yamamoto, and A. Yagi, "Exponential attractors for non-autonomous dissipative system," Journal of the Mathematical Society of Japan, vol. 63, no. 2, pp. 647-673, 2011.

[16] X. Han, "Exponential attractors for lattice dynamical systems in weighted spaces," Discrete and Continuous Dynamical Systems A, vol. 31, no. 2, pp. 445-467, 2011.

[17] J. A. Langa, A. Miranville, and J. Real, "Pullback exponential attractors," Discrete and Continuous Dynamical Systems A, vol. 26, no. 4, pp. 1329-1357, 2010.

[18] M. Efendiev, A. Miranville, and S. Zelik, "Exponential attractors for a nonlinear reaction-diffusion system in $\mathbb{R}^{3}$," Comptes Rendus de l'Académie des Sciences, vol. 330, no. 8, pp. 713-718, 2000.

[19] M. Efendiev, A. Miranville, and S. Zelik, "Global and exponential attractors for nonlinear reaction-diffusion systems in unbounded domains," Proceedings of the Royal Society of Edinburgh A, vol. 134, no. 2, pp. 271-315, 2004.

[20] S. Zhou and X. Han, "Pullback exponential attractors for non-autonomous lattice systems," Journal of Dynamics and Differential Equations, vol. 24, no. 3, pp. 601-631, 2012.

[21] X. Fan and H. Yang, "Exponential attractor and its fractal dimension for a second order lattice dynamical system," Journal of Mathematical Analysis and Applications, vol. 367, no. 2, pp. 350-359, 2010.

[22] X. Wang, S. Li, and D. Xu, "Random attractors for secondorder stochastic lattice dynamical systems," Nonlinear Analysis. Theory, Methods \& Applications A, vol. 72, no. 1, pp. 483-494, 2010.

[23] S. Zhou, "Attractors and approximations for lattice dynamical systems," Journal of Differential Equations, vol. 200, no. 2, pp. 342-368, 2004.

[24] A. Pazy, Semigroups of Linear Operator and Applications to Partial Differential Equations, Springer, New York, NY, USA, 2007. 


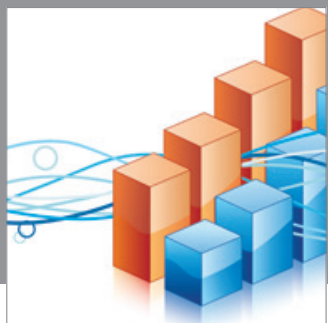

Advances in

Operations Research

mansans

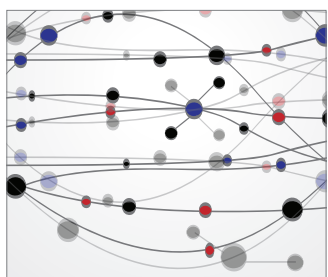

The Scientific World Journal
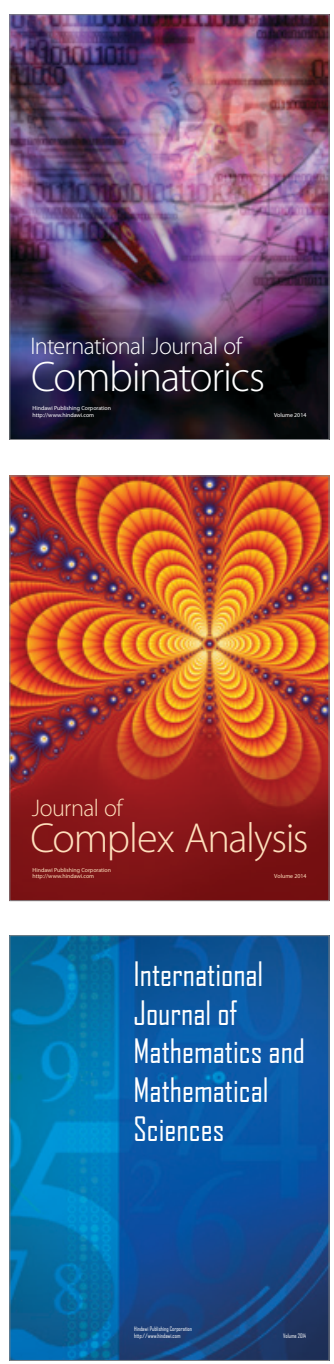
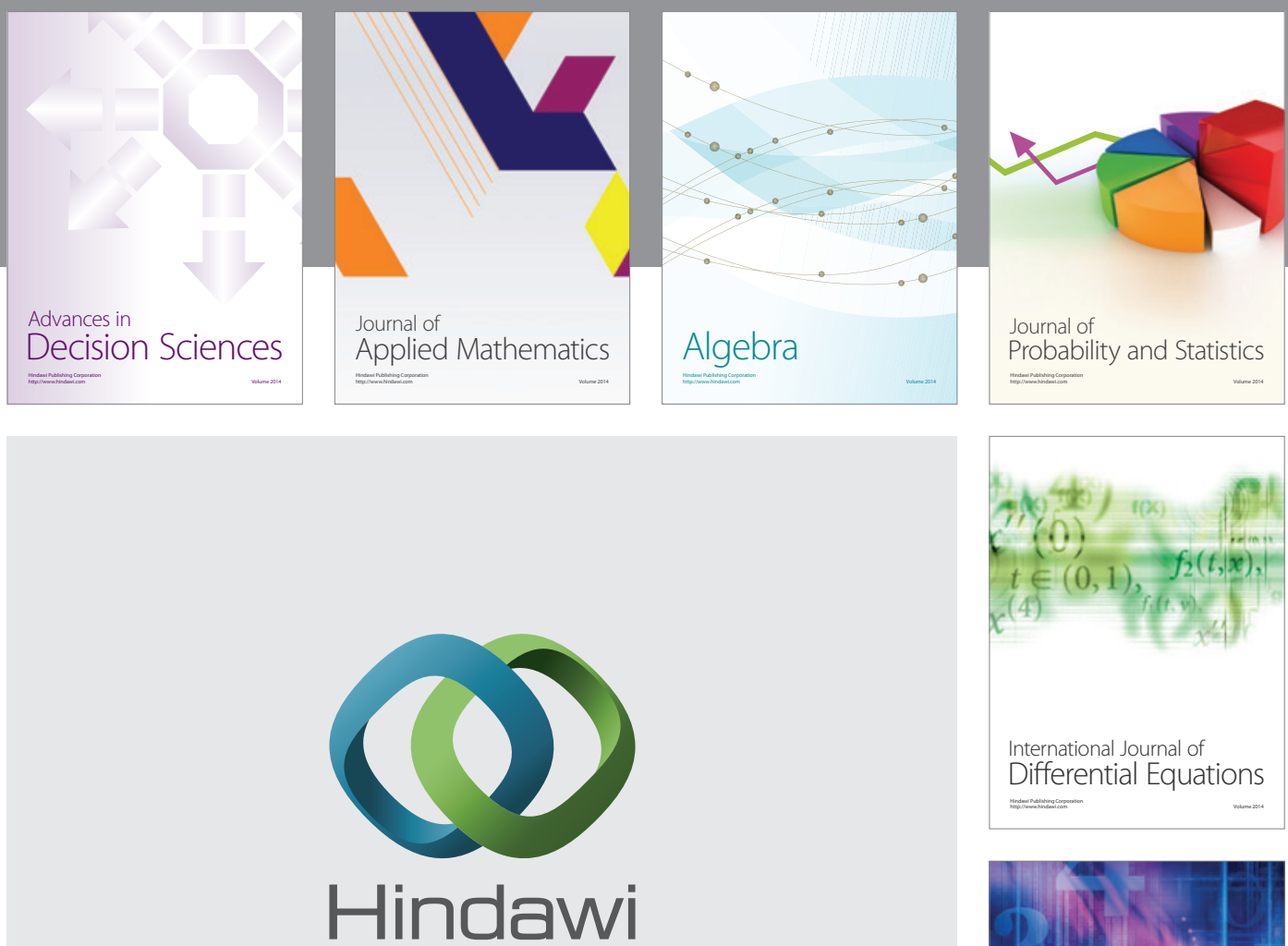

Submit your manuscripts at http://www.hindawi.com
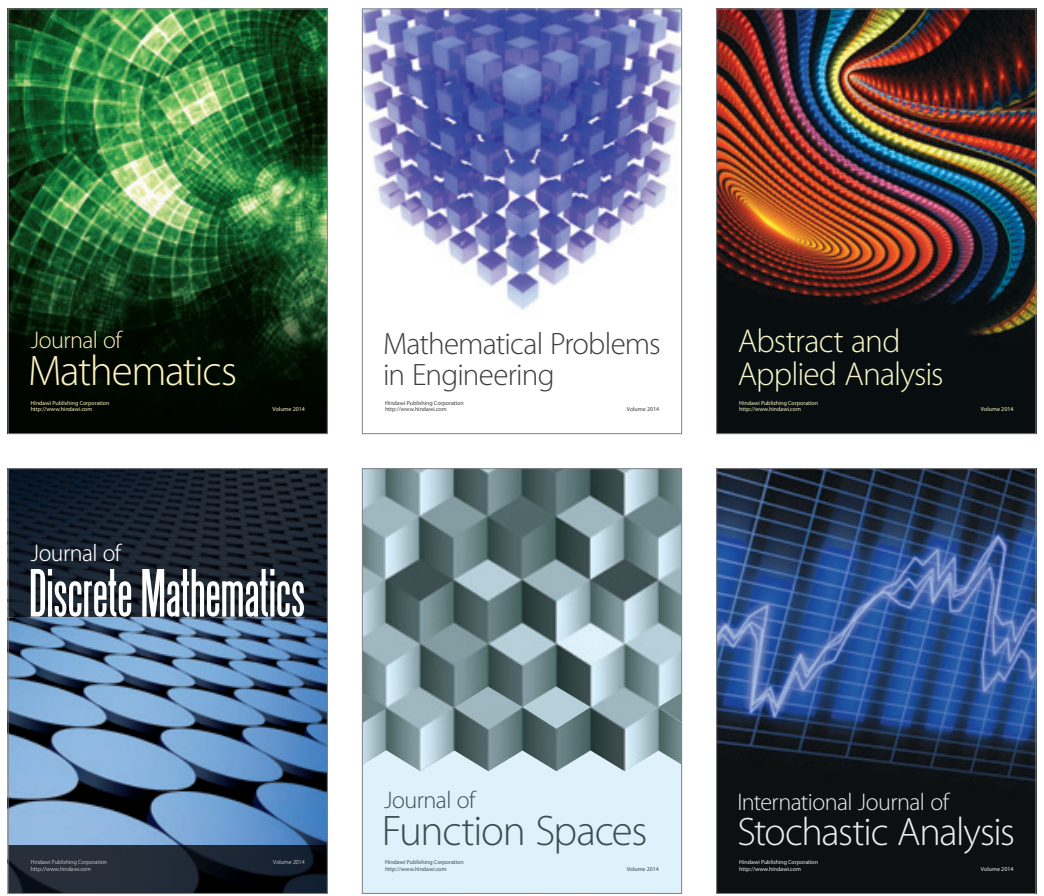

Journal of

Function Spaces

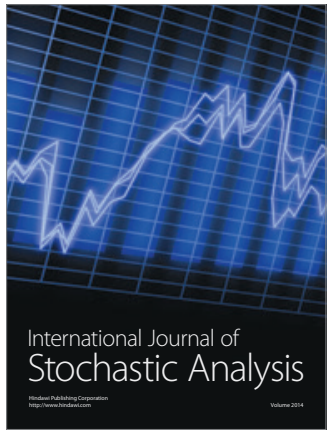

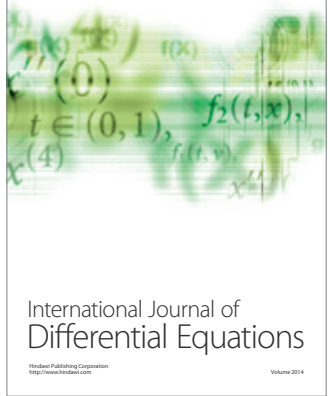
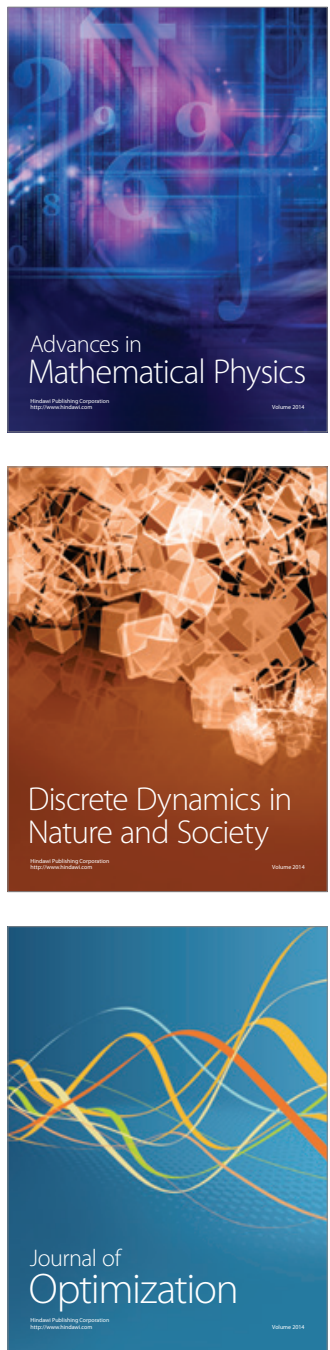